\title{
Fractional singular Sturm-Liouville operator for Coulomb potential
}

\author{
Erdal Bas ${ }^{*}$ and Funda Metin
}

\author{
"Correspondence: \\ erdalmat@yahoo.com \\ Department of Mathematics, \\ Faculty of Science, Firat University, \\ Elazig, 23119, Turkey
}

\begin{abstract}
In this paper, we define a fractional singular Sturm-Liouville operator having Coulomb potential of type $\frac{A}{x}$. Our main issue is to investigate the spectral properties for the operator. Furthermore, we prove new results according to the fractional singular Sturm-Liouville problem.

MSC: $26 \mathrm{~A} 33 ; 34 \mathrm{~A} 08$

Keywords: fractional; Sturm-Liouville; Caputo; Coulomb; singular; spectral
\end{abstract}

\section{Introduction}

The idea of generalizing differential operators to a non-integer order, especially to the order $\frac{1}{2}$, appeared in the correspondence of L' Hospital and Leibniz in 1695. Later Fourier, Euler and Laplace were among the many that studied fractional calculus and mathematical consequences. They found, using their own notation and methodology, definitions that were suitable for the concept of non-integer order integral or derivative. The most famous of these definitions that have been popularized in the world of fractional calculus are the Riemann-Liouville, Grunwald-Letnikov and Caputo definitions. Fractional differential equations have been of increasing importance for the last years due to their diverse applications in the fields of physics, chemistry, biology, economics, control theory, optical systems, etc. [1-7]. But why is fractional calculus important? Until recent times, fractional calculus was considered as a rather esoteric mathematical theory without applications, but in the last decade there was an explosion of research activity on the application of fractional calculus to very diverse scientific fields ranging from the physics of diffusion and advection phenomena, to control systems and to finance and economics. Indeed, at present, applications and/or activities related to fractional calculus have appeared in at least the following fields [8]:

- Fractional control of engineering systems;

- Advancement of calculus of variations and optimal control to fractional dynamic systems;

- Analytical and numerical tools and techniques;

- Fundamental explorations of the mechanical, electrical, and thermal constitutive relations and other properties of various engineering materials such as viscoelastic polymers, foams, gels, and animal tissues;

- Fundamental understanding of wave and diffusion phenomenon, their measurements and verifications, including applications to plasma physics (such as diffusion in Tokamak);

○2013 Bas and Metin; licensee Springer. This is an Open Access article distributed under the terms of the Creative Commons Attribution License (http://creativecommons.org/licenses/by/2.0), which permits unrestricted use, distribution, and reproduction in any medium, provided the original work is properly cited. 
- Bioengineering and biomedical applications;

- Thermal modeling of engineering systems such as brakes and machine tools;

- Image and signal processing.

Many important results of the existence of solutions of various classes of fractional differential equations were given by Oldham, Spainer, Kilbas, Marichev, Gorenflo, Miller, Podlubny, Baleanu, Agarwal, Ross, Srivastava etc. Most of the mathematical theory applicable to the study of fractional calculus was developed prior to the turn of the twentieth century [4-19]. For last centuries, the theory of fractional derivatives developed mainly as a pure theoretical field of mathematics useful only for mathematicians.

Furthermore, Sturm-Liouville problems have been known since 1836. The importance of mathematics arises from the study of problems in the real world. The spectral characteristics are spectra, spectral functions, scattering data, norming constants for using Sturm-Liouville problems. The concept of Sturm-Liouville problems plays an important role in mathematics and physics. The progress in applied mathematics was obtained by the extension and development of many important analytical approaches and methods. There have been numerous studies that focus on this problem since then [19-27]. Nowadays, new approaches to fractional Sturm-Liouville problem are produced [17-20].

In this paper, we deal with the fractional Sturm-Liouville operator with Coulomb potential. Before giving the main results for the singular fractional operator, we give some fundamental physical properties of the Sturm-Liouville operator with Coulomb potential. Learning about the motion of electrons moving under the Coulomb potential is of significance in quantum theory. Solving these types of problems provides us with finding energy levels of not only hydrogen atom but also of single valance electron atoms such as sodium. For the Coulomb potential is given by $U=\frac{-e^{2}}{r}$, where $r$ is the radius of the nucleus, $e$ is electronic charge. According to this, we use time-dependent Schrödinger equation

$$
i \hbar \frac{\partial \Psi}{\partial t}=-\frac{\hbar^{2}}{2 m} \frac{\partial^{2} \Psi}{\partial x^{2}}+U(x, y, z) \Psi, \quad \int_{R^{3}}|\Psi|^{2} d x d y d z=1,
$$

where $\Psi$ is the wave function, $\hbar$ is Planck's constant and $m$ is the mass of electron. In this equation, if the Fourier transform is applied

$$
\widetilde{\Psi}=\frac{1}{\sqrt{2 \pi}} \int_{-\infty}^{\infty} e^{-i \lambda t} \Psi d t
$$

it will convert to energy equation dependent on the situation as follows:

$$
\frac{\hbar^{2}}{2 m} \nabla^{2} \widetilde{\Psi}+\widetilde{U} \widetilde{\Psi}=E \widetilde{\Psi}
$$

Therefore, energy equation in the field with Coulomb potential becomes

$$
-\frac{\hbar^{2}}{2 m} \nabla^{2} \widetilde{\Psi}+\left(E+\frac{e^{2}}{r}\right) \widetilde{\Psi}=0 .
$$

If this hydrogen atom is substituted to other potential area, then energy equation becomes

$$
-\frac{\hbar^{2}}{2 m} \nabla^{2} \widetilde{\Psi}+\left(E+\frac{e^{2}}{r}+q(x, y, z)\right) \widetilde{\Psi}=0 .
$$


If we make the necessary transformation, then we can get the Sturm-Liouville equation with Coulomb potential

$$
-y^{\prime \prime}+\left[\frac{A}{x}+q(x)\right] y=\lambda y
$$

where $\lambda$ is a parameter which corresponds to the energy and $C$ is a constant [28].

We should note that Klimek and Agrawal [20] defined a fractional Sturm-Liouville operator, introduced a regular fractional Sturm-Liouville problem and investigated the properties of eigenfunctions and eigenvalues of the operator. In this paper, our aim is to introduce a singular fractional Sturm-Liouville problem with Coulomb potential and prove spectral properties of spectral data for the operator. We also show that the fractional SturmLiouville operator with Coulomb potential is self adjoint, in addition to [20].

Let us give some important necessary data that will be used in the main results.

\section{Preliminaries}

Definition 1 [4] Let $0<\alpha \leq 1$. The left-sided and respectively right-sided RiemannLiouville integrals of order $\alpha$ are given by the formulas

$$
\begin{aligned}
& \left(I_{a,+}^{\alpha} f\right)(x)=\frac{1}{\Gamma(\alpha)} \int_{a}^{x}(x-s)^{\alpha-1} f(s) d s, \quad x>a, \\
& \left(I_{b, f}^{\alpha} f\right)(x)=\frac{1}{\Gamma(\alpha)} \int_{x}^{b}(s-x)^{\alpha-1} f(s) d s, \quad x<b,
\end{aligned}
$$

where $\Gamma$ denotes the gamma function.

Definition 2 [4] Let $0<\alpha \leq 1$. The left-sided and respectively right-sided RiemannLiouville derivatives of order $\alpha$ are defined as

$$
\begin{aligned}
& \left(D_{a,+}^{\alpha} f\right)(x)=D\left(I_{a,+}^{1-\alpha} f\right)(x), \quad x>a, \\
& \left(D_{b,-}^{\alpha} f\right)(x)=-D\left(I_{b,-}^{1-\alpha} f\right)(x), \quad x<b .
\end{aligned}
$$

Analogous formulas yield the left- and right-sided Caputo derivatives of order $\alpha$ :

$$
\begin{aligned}
& \left({ }^{C} D_{a,+}^{\alpha} f\right)(x)=\left(I_{a,+}^{1-\alpha} D f\right)(x), \quad x>a, 0<\alpha \leq 1, \\
& \left({ }^{C} D_{b,-}^{\alpha} f\right)(x)=\left(I_{b,-}^{1-\alpha}(-D) f\right)(x), \quad x<b, 0<\alpha \leq 1 .
\end{aligned}
$$

Definition 3 [7] The general function ${ }_{p} \Psi_{q}(z)$ is defined for $z \in \mathbb{C}, a_{l}, b_{j} \in \mathbb{C}, \alpha_{l}, \beta_{j} \in \mathbb{R}$ $(l=1, \ldots, p ; j=1, \ldots, q)$ by the series

$$
\begin{aligned}
{ }_{p} \Psi_{q}(z) & \left.={ }_{p} \Psi_{q}\left[\begin{array}{c}
\left(a_{1}, \alpha_{1}\right)_{1, p} \mid \\
\left(b_{1}, \beta_{1}\right)_{1, q}
\end{array}\right] z\right] \\
& =\sum_{k=0}^{\infty} \frac{\prod_{l=1}^{p} \Gamma\left(a_{l}+\alpha_{l} k\right)}{\prod_{j=1}^{q} \Gamma\left(b_{j}+\beta_{j} k\right)} \frac{z^{k}}{k !} .
\end{aligned}
$$


This general Wright function was investigated by Fox who presented its asymptotic expansion for large values of the argument $z$ under the condition

$$
\sum_{j=1}^{q} \beta_{j}-\sum_{l=1}^{p} \alpha_{l}>1 .
$$

If these conditions are satisfied, the series in (7) is convergent for any $z \in \mathbb{C}$.

Theorem 4 [7] Let $a_{l}, b_{j} \in \mathbb{C}$, and $\alpha_{l}, \beta_{j} \in \mathbb{R}(l=1, \ldots, p ; j=1, \ldots, q)$, and let

$$
\begin{aligned}
& \Delta=\sum_{j=1}^{q} \beta_{j}-\sum_{l=1}^{p} \alpha_{l}, \\
& \delta=\prod_{l=1}^{p}\left|\alpha_{l}\right|^{-\alpha_{l}} \prod_{j=1}^{q}\left|\beta_{j}\right|^{\beta_{j}}, \\
& \mu=\sum_{j=1}^{q} b_{j}-\sum_{l=1}^{p} a_{l}+\frac{p-q}{2} .
\end{aligned}
$$

(I) If $\Delta>-1$, then the series in (7) is absolutely convergent for all $z \in \mathbb{C}$.

(II) If $\Delta=-1$, then the series in (7) is absolutely convergent for $|z|<\delta$ and for $|z|=\delta$ and $\Re(\mu)>1 / 2$.

Theorem 5 [29] Let $(X, d)$ be a non-empty complete metric space. Let $T: X \rightarrow X$ be a contraction mapping on $X$, i.e., there is a nonnegative real number $q<1$ such that

$$
d(T(x), T(y)) \leq q d(x, y)
$$

for all $x, y$ in $X$. Then the map $T$ admits one and only one fixed-point $x^{*}$ in $x$ (this means $\left.T\left(x^{*}\right)=x^{*}\right)$. Furthermore, this fixed point can be found as follows starting with an arbitrary element $x_{0}$ in $X$ and defining an iterative sequence by $x_{n}=T\left(x_{n-1}\right)$ for $n=1,2,3, \ldots$. This sequence converges, and its limit is $x^{*}$. The following inequality describes the speed of convergence:

$$
d\left(x^{*}, x_{n}\right) \leq \frac{q_{n}}{1-q} d\left(x_{1}, x_{0}\right) .
$$

Equivalently,

$$
d\left(x^{*}, x_{n+1}\right) \leq \frac{q}{1-q} d\left(x_{n+1}, x_{n}\right)
$$

and

$$
d\left(x^{*}, x_{n+1}\right) \leq q d\left(x^{*}, x_{n}\right) .
$$

Any such value of $q$ is called a Lipschitz constant for $T$, and the smallest one is sometimes called 'the best Lipschitz constant' of T. 
Property 6 The fractional differential operators defined in (3)-(6) satisfy the following identities:

$$
\int_{a}^{b} f(x) D_{b,-}^{\alpha} g(x) d x=\int_{a}^{b} g(x)^{C} D_{a,+}^{\alpha} f(x) d x-\left.f(x) I_{b,-}^{1-\alpha} g(x)\right|_{a} ^{b} ;
$$

on the other hand,

$$
\begin{aligned}
& \int_{a}^{b} f(x) D_{b,-}^{\alpha} g(x)^{C} D_{a,+}^{\alpha} k(x) d x \\
& \quad \int_{a}^{b} g(x)^{C} D_{a,+}^{\alpha} f(x){ }^{C} D_{a,+}^{\alpha} k(x) d x-\left.f(x) I_{b,-}^{1-\alpha} g(x){ }^{C} D_{a,+}^{\alpha} k(x)\right|_{a} ^{b}, \\
& \int_{a}^{b} f(x) D_{a,+}^{\alpha} g(x) d x=\int_{a}^{b} g(x)^{C} D_{b,-}^{\alpha} f(x) d x+\left.f(x) I_{a,+}^{1-\alpha} g(x)\right|_{a} ^{b} .
\end{aligned}
$$

Property 7 Assume $\alpha \in(0,1], \beta>\alpha$ and $f \in C[a, b]$. Then the following relations

$$
\begin{aligned}
& D_{a,+}^{\alpha} I_{a,+}^{\alpha} f(x)=f(x), \\
& D_{b,-}^{\alpha} I_{b,-}^{\alpha} f(x)=f(x), \\
& D_{a,+}^{\alpha} I_{a,+}^{\beta} f(x)=I_{a,+}^{\beta-\alpha} f(x), \\
& D_{b,-}^{\alpha} I_{b, f}^{\beta} f(x)=I_{b,-}^{\beta-\alpha} f(x), \\
& { }^{C} D_{a,+}^{\alpha} I_{a,+}^{\alpha} f(x)=f(x), \\
& { }^{C} D_{b,-}^{\alpha} I_{b,-}^{\alpha} f(x)=f(x)
\end{aligned}
$$

hold for any $x \in[a, b]$. Furthermore, the integral operators defined in (1), (2) satisfy the following semi-group properties:

$$
I_{a,+}^{\alpha} I_{a,+}^{\beta}=I_{a,+}^{\alpha+\beta}, \quad I_{b,-}^{\alpha} I_{b,-}^{\beta}=I_{b,-}^{\alpha+\beta} .
$$

Now, let us take up a fractional singular Sturm-Liouville problem for Coulomb potential.

\section{Main results}

Let us denote a fractional Sturm-Liouville problem for Coulomb potential with the differential part containing the left- and right-sided derivatives. Let us use the form of the integration by parts formulas (8), (9) for this new approximation. Main properties of eigenfunctions and eigenvalues in the theory of classical Sturm-Liouville problems are related to the integration by parts formula for the first-order derivatives. In the corresponding fractional version, we note that both left and right derivatives appear and the essential pairs are the left Riemann-Liouville derivative with the right Caputo derivative and the right Riemann-Liouville derivative with the left Caputo one.

Let $\alpha \in(0,1)$. A fractional Sturm-Liouville operator for Coulomb potential is written as

$$
\mathcal{L}_{\alpha[C]}=D_{\pi,-}^{\alpha} p(x)^{C} D_{0,+}^{\alpha}+\left(\frac{A}{x}+q(x)\right)
$$


Consider the fractional Sturm-Liouville equation for Coulomb potential

$$
\mathcal{L}_{\alpha[C]} y_{\lambda}(x)+\lambda w_{\alpha}(x) y_{\lambda}(x)=0
$$

where $p(x) \neq 0, w_{\alpha}(x)>0 \forall x \in(0, \pi], w_{\alpha}(x)$ is a weight function and $p, q$ are real-valued continuous functions in the interval $(0, \pi]$ and $\frac{y_{\lambda}(x)}{x} \in C[0, \pi]$. The boundary conditions for the operator $\mathcal{L}$ are the following:

$$
\begin{aligned}
& y_{\lambda}(0)=0, \\
& c_{1} y_{\lambda}(\pi)-c_{2} I_{\pi,-}^{1-\alpha} p(\pi)^{C} D_{0,+}^{\alpha} y_{\lambda}(\pi)=0,
\end{aligned}
$$

where $c_{1}^{2}+c_{2}^{2} \neq 0$. Fractional boundary value problem (12)-(14) is a fractional SturmLiouville problem for Coulomb potential.

Theorem 8 Fractional Sturm-Liouville operator for Coulomb potential is self-adjoint $(0, \pi]$.

Proof Let us consider the following equation:

$$
\begin{aligned}
\left\langle\mathcal{L}_{\alpha[C]} \varphi, \phi\right\rangle= & \int_{0}^{\pi} \mathcal{L}_{\alpha[C]} \varphi(x) \phi(x) d x \\
= & \int_{0}^{\pi} \phi(x)\left[D_{\pi,-}^{\alpha} p(x)^{C} D_{0,+}^{\alpha} \varphi(x)+\left(\frac{A}{x}+q(x)\right) \varphi(x)\right] d x \\
= & \int_{0}^{\pi} \phi(x) D_{\pi,-}^{\alpha} p(x)^{C} D_{0,+}^{\alpha} \varphi(x) d x \\
& +\int_{0}^{\pi}\left(\frac{A}{x}+q(x)\right) \varphi(x) \phi(x) d x .
\end{aligned}
$$

By means of property (8) and boundary conditions (13)-(14), we obtain the identity

$$
\begin{aligned}
\left\langle\mathcal{L}_{\alpha[C]} \varphi, \phi\right\rangle= & \int_{0}^{\pi} p(x){ }^{C} D_{0,+}^{\alpha} \phi(x){ }^{C} D_{0,+}^{\alpha} \varphi(x) d x-\left.\phi(x) I_{\pi,-}^{1-\alpha} p(x){ }^{C} D_{0,+}^{\alpha} \varphi(x)\right|_{0} ^{\pi} \\
& +\int_{0}^{\pi}\left(\frac{A}{x}+q(x)\right) \varphi(x) \phi(x) d x \\
= & \int_{0}^{\pi} p(x)^{C} D_{0,+}^{\alpha} \phi(x)^{C} D_{0,+}^{\alpha} \varphi(x) d x-\frac{C_{1}}{c_{2}} \varphi(\pi) \phi(\pi) \\
& +\int_{0}^{\pi}\left(\frac{A}{x}+q(x)\right) \varphi(x) \phi(x) d x .
\end{aligned}
$$

On the other hand, by performing similar necessary operations, we obtain

$$
\begin{aligned}
\left\langle\varphi, \mathcal{L}_{\alpha[C]} \phi\right\rangle= & \int_{0}^{\pi} p(x)^{C} D_{0,+}^{\alpha} \varphi(x)^{C} D_{0,+}^{\alpha} \phi(x) d x-\frac{c_{1}}{c_{2}} \varphi(\pi) \phi(\pi) \\
& +\int_{0}^{\pi}\left(\frac{A}{x}+q(x)\right) \phi(x) \varphi(x) d x .
\end{aligned}
$$


The right-hand sides of equations (15) and (16) are equal, hence we may see that the lefthand sides are equal, that is,

$$
\left\langle\mathcal{L}_{\alpha[C]} \varphi, \phi\right\rangle=\left\langle\varphi, \mathcal{L}_{\alpha[C]} \phi\right\rangle .
$$

The proof is completed.

Theorem 9 The eigenvalues of a fractional singular Sturm-Liouville operator with Coulomb potential (12)-(14) are real.

Proof Let us observe that the following relation results from property (8)

$$
\begin{aligned}
\int_{0}^{\pi} f(x) \mathcal{L}_{\alpha[C]} g(x) d x= & \int_{0}^{\pi} p(x)^{C} D_{0,+}^{\alpha} f(x)^{C} D_{0,+}^{\alpha} g(x) d x-\left.f(x) I_{\pi,-}^{1-\alpha} p(x)^{C} D_{0,+}^{\alpha} g(x)\right|_{0} ^{\pi} \\
& +\int_{0}^{\pi}\left(\frac{A}{x}+q(x)\right) g(x) f(x) d x .
\end{aligned}
$$

Suppose that $\lambda$ is the eigenvalue for (12)-(14) corresponding to the eigenfunction complex conjugate $\bar{y}$, then the following equalities are satisfied:

$$
\begin{aligned}
& \mathcal{L}_{\alpha[C]} \bar{y}(x)+\bar{\lambda} w_{\alpha}(x) \bar{y}(x)=0, \\
& \bar{y}(0)=0, \\
& c_{1} \bar{y}(\pi)-c_{2} I_{\pi,-}^{1-\alpha} p(\pi)^{C} D_{0,+}^{\alpha} \bar{y}(\pi)=0,
\end{aligned}
$$

where $c_{1}^{2}+c_{2}^{2} \neq 0$. We multiply equation (12) by function $\bar{y}$ and (18) by function $y$, respectively, and subtract:

$$
(\lambda-\bar{\lambda}) w_{\alpha}(x) y(x) \bar{y}(x)=y(x) \mathcal{L}_{\alpha[C]} \bar{y}(x)-\bar{y}(x) \mathcal{L}_{\alpha[C]} y(x) .
$$

Now, we integrate over interval $(0, \pi]$ and applying relation (17), we note that the righthand side of the integrated equality contains only boundary terms:

$$
\begin{aligned}
& (\lambda-\bar{\lambda}) \int_{0}^{\pi} w_{\alpha}(x) y(x) \bar{y}(x) d x \\
& =\int_{0}^{\pi} y(x) \mathcal{L}_{\alpha[C]} \bar{y}(x) d x-\int_{0}^{\pi} \bar{y}(x) \mathcal{L}_{\alpha[C]} y(x) d x \\
& =\int_{0}^{\pi} p(x)^{C} D_{0,+}^{\alpha} y(x)^{C} D_{0,+}^{\alpha} \bar{y}(x) d x-\left.y(x) I_{\pi,-}^{1-\alpha} p(x){ }^{C} D_{0,+}^{\alpha} \bar{y}(x)\right|_{0} ^{\pi} \\
& \quad-\int_{0}^{\pi} p(x)^{C} D_{0,+}^{\alpha} \bar{y}(x)^{C} D_{0,+}^{\alpha} y(x) d x+\left.\bar{y}(x) I_{\pi,-}^{1-\alpha} p(x){ }^{C} D_{0,+}^{\alpha} y(x)\right|_{0} ^{\pi}, \\
& (\lambda-\bar{\lambda}) \int_{0}^{\pi} w_{\alpha}(x)|y(x)|^{2} d x=-\left.y(x) I_{\pi,-}^{1-\alpha} p(x)^{C} D_{0,+}^{\alpha} \bar{y}(x)\right|_{\pi}+\left.y(x) I_{\pi,-}^{1-\alpha} p(x)^{C} D_{0,+}^{\alpha} \bar{y}(x)\right|_{0} \\
& \quad+\left.\bar{y}(x) I_{\pi,-}^{1-\alpha} p(x)^{C} D_{0,+}^{\alpha} y(x)\right|_{\pi}-\left.\bar{y}(x) I_{\pi,-}^{1-\alpha} p(x)^{C} D_{0,+}^{\alpha} y(x)\right|_{0} .
\end{aligned}
$$

By virtue of boundary conditions (13), (14), (19), (20), we find

$$
(\lambda-\bar{\lambda}) \int_{0}^{\pi} w_{\alpha}(x)|y(x)|^{2} d x=0,
$$


and because $y$ is a non-trivial solution and $w_{\alpha}(x)>0$, it easily seen that $\lambda=\bar{\lambda}$. This proves the theorem.

Theorem 10 The eigenfunctions corresponding to distinct eigenvalues of the fractional Sturm-Liouville problem with Coulomb potential (12)-(14) are orthogonal weight function $w_{\alpha}$ on $(0, \pi]$, that is,

$$
\int_{0}^{\pi} w_{\alpha}(x) y_{\lambda_{1}}(x) y_{\lambda_{2}}(x) d x=0, \quad \lambda_{1} \neq \lambda_{2} .
$$

Proof We have by assumptions the fractional singular Sturm-Liouville problem with Coulomb potential fulfilled by two different eigenvalues $\left(\lambda_{1}, \lambda_{2}\right)$ and the respective eigenfunctions $\left(y_{\lambda_{1}}, y_{\lambda_{2}}\right)$ :

$$
\begin{aligned}
& \mathcal{L}_{\alpha[C]} y_{\lambda_{1}}(x)+\lambda_{1} w_{\alpha}(x) y_{\lambda_{1}}(x)=0, \\
& y_{\lambda_{1}}(x)=0, \\
& c_{1} y_{\lambda_{1}}(\pi)-c_{2} I_{\pi,-}^{1-\alpha} p(\pi)^{C} D_{0,+}^{\alpha} y_{\lambda_{1}}(\pi)=0, \\
& \mathcal{L}_{\alpha[C]} y_{\lambda_{2}}(x)+\lambda_{2} w_{\alpha}(x) y_{\lambda_{2}}(x)=0, \\
& y_{\lambda_{2}}(x)=0, \\
& c_{1} y_{\lambda_{2}}(\pi)-c_{2} I_{\pi,-}^{1-\alpha} p(\pi)^{C} D_{0,+}^{\alpha} y_{\lambda_{2}}(\pi)=0 .
\end{aligned}
$$

We multiply equation (21) by function $y_{\lambda_{2}}$ and (24) by function $y_{\lambda_{1}}$, respectively, and subtract:

$$
\left(\lambda_{1}-\lambda_{2}\right) w_{\alpha}(x) y_{\lambda_{1}} y_{\lambda_{2}}=y_{\lambda_{1}} \mathcal{L}_{\alpha[C]} y_{\lambda_{2}}-y_{\lambda_{2}} \mathcal{L}_{\alpha[C]} y_{\lambda_{1}} .
$$

Integrating over the interval $(0, \pi]$ and applying relation (17), we note that the right-hand side of the integrated equality contains only boundary terms:

$$
\begin{aligned}
\left(\lambda_{1}-\right. & \left.\lambda_{2}\right) \int_{0}^{\pi} w_{\alpha}(x) y_{\lambda_{1}}(x) y_{\lambda_{2}}(x) d x \\
= & \int_{0}^{\pi} y_{\lambda_{1}}(x) \mathcal{L}_{\alpha[C]} y_{\lambda_{2}}(x) d x-\int_{0}^{\pi} y_{\lambda_{2}}(x) \mathcal{L}_{\alpha[C]} y_{\lambda_{1}}(x) d x \\
= & \int_{0}^{\pi} y_{\lambda_{1}}(x)\left[D_{\pi,-}^{\alpha} p(x)^{C} D_{0,+}^{\alpha} y_{\lambda_{2}}(x)+\left(\frac{A}{x}+q(x)\right) y_{\lambda_{2}}(x)\right] d x \\
& -\int_{0}^{\pi} y_{\lambda_{2}}(x)\left[D_{\pi,-}^{\alpha} p(x)^{C} D_{0,+}^{\alpha} y_{\lambda_{1}}(x)+\left(\frac{A}{x}+q(x)\right) y_{\lambda_{1}}(x)\right] d x \\
= & \int_{0}^{\pi} p(x)^{C} D_{0,+}^{\alpha} y_{\lambda_{1}}(x)^{C} D_{0,+}^{\alpha} y_{\lambda_{2}}(x) d x-\left.y_{\lambda_{1}}(x) I_{\pi,-}^{1-\alpha} p(x)^{C} D_{0,+}^{\alpha} y_{\lambda_{2}}(x)\right|_{0} ^{\pi} \\
& -\int_{0}^{\pi} p(x)^{C} D_{0,+}^{\alpha} y_{\lambda_{2}}(x)^{C} D_{0,+}^{\alpha} y_{\lambda_{1}}(x) d x+\left.y_{\lambda_{2}}(x) I_{\pi,-}^{1-\alpha} p(x)^{C} D_{0,+}^{\alpha} y_{\lambda_{1}}(x)\right|_{0} ^{\pi}, \\
\left(\lambda_{1}-\right. & \left.\lambda_{2}\right) \int_{0}^{\pi} w_{\alpha}(x) y_{\lambda_{1}}(x) y_{\lambda_{2}}(x) d x \\
= & -\left.y_{\lambda_{1}}(x) I_{\pi,-}^{1-\alpha} p(x)^{C} D_{0,+}^{\alpha} y_{\lambda_{2}}(x)\right|_{0} ^{\pi}+\left.y_{\lambda_{2}}(x) I_{\pi,-}^{1-\alpha} p(x)^{C} D_{0,+}^{\alpha} y_{\lambda_{1}}(x)\right|_{0} ^{\pi} .
\end{aligned}
$$


Using the boundary conditions (22), (23), (25), (26), we find that

$$
\left(\lambda_{1}-\lambda_{2}\right) \int_{0}^{\pi} w_{\alpha}(x) y_{\lambda_{1}}(x) y_{\lambda_{2}}(x) d x=0,
$$

where $\lambda_{1} \neq \lambda_{2}$.

Remark Let us now give certain auxiliary functions. Because we use the functions, the first of them is as follows:

$$
I_{0,+}^{\alpha} \frac{(\pi-x)^{\alpha-1}}{\Gamma(\alpha)}=(\pi-0)^{\alpha-1}(x-0)^{\alpha}{ }_{1} \Psi_{2}\left[\begin{array}{cc}
(1,1) & \\
(\alpha,-1) & (\alpha+1,1)
\end{array} \mid-\frac{x-0}{\pi-0}\right],
$$

where ${ }_{1} \Psi_{2}$ is the Fox-Wright function [7].

$$
{ }_{1} \Psi_{2}\left[\begin{array}{cc}
\left(a_{1}, \alpha_{1}\right) & \\
\left(b_{1}, \beta_{1}\right) & \left(b_{2}, \beta_{2}\right)
\end{array} \mid z\right]=\sum_{k=0}^{\infty} \frac{\Gamma\left(a_{1}+\alpha_{1} k\right)}{\Gamma\left(b_{1}+\beta_{1} k\right) \Gamma\left(b_{2}+\beta_{2} k\right)} \frac{z^{k}}{k !},
$$

the properties of the function are determined by the parameters

$$
\begin{aligned}
& \Delta=\beta_{1}+\beta_{2}-\alpha_{1}=-1, \\
& \delta=\left|\alpha_{1}\right|^{-\alpha_{1}}\left|\beta_{1}\right|^{\beta_{1}}\left|\beta_{2}\right|^{\beta_{2}}=1, \\
& \mu=b_{1}+b_{2}-\alpha_{1}+\frac{1-2}{2}=2 \alpha-\frac{1}{2} .
\end{aligned}
$$

Considering Theorem 4 , we note that this function is continuous in $(0, \pi]$ when order $\alpha>$ $1 / 2$, i.e., $\mu>1 / 2$. For $0<\alpha \leq 1 / 2$, it is discontinuous at end $x=\pi$. The explicitly calculated function allows us to estimate the second component of the stationary function $\phi_{0}$ of the differential part of the Sturm-Liouville operator

$$
D_{\pi,-}^{\alpha} p(x)^{C} D_{0,+}^{\alpha} \phi_{0}(x)=0
$$

which looks as follows:

$$
\phi_{0}(x)=\xi_{1}+\xi_{2} I_{0,+}^{\alpha} \frac{(\pi-x)^{\alpha-1}}{\Gamma(\alpha) p(x)}=\xi_{1}+\xi_{2} \psi(\alpha, 0, x) .
$$

The next function is the following integral:

$$
\begin{aligned}
\varphi(x) & =I_{0,+}^{\alpha} I_{\pi,-}^{\alpha} 1=I_{0,+}^{\alpha} \frac{(\pi-x)^{\alpha}}{\Gamma(\alpha+1)} \\
& =(\pi-0)^{\alpha}(x-0)^{\alpha} \times{ }_{1} \Psi_{2}\left[\begin{array}{cc}
(1,1) & \\
(\alpha+1,-1) & (\alpha+1,1)
\end{array} \mid-\frac{x-0}{\pi-0}\right] .
\end{aligned}
$$

Again, using Theorem 4 and calculating parameters according to (27), we get

$$
\Delta=-1, \quad \delta=1, \quad \mu=2 \alpha+\frac{1}{2} .
$$


We conclude

$$
\alpha>0 \quad \Longrightarrow \quad \mu>\frac{1}{2}
$$

and the obtained Fox-Wright function (29) is continuous in the interval $(0, \pi]$ for any positive order $\alpha$.

Lemma 11 Let $\alpha>1 / 2$ and denote

$$
\begin{aligned}
& Y_{\lambda}(y)=\left(\frac{A}{x}+q(x)\right) y_{\lambda}(x)+\lambda w_{\alpha} y_{\lambda}(x), \\
& \tilde{\Delta}=-c_{2}+c_{1} \psi(\alpha, 0, \pi) .
\end{aligned}
$$

Assume $\tilde{\Delta} \neq 0$. Then, on the $C[0, \pi]$-space, (12)-(14) is equivalent to the integral equation

$$
y_{\lambda}(x)=-I_{0,+}^{\alpha} \frac{1}{p(x)} I_{\pi,-}^{\alpha} Y_{\lambda}(y)+\left.A(x)\left(I_{0,+}^{\alpha} \frac{1}{p(x)} I_{\pi,-}^{\alpha} Y_{\lambda}(y)\right)\right|_{x=\pi},
$$

where the coefficient $A(x)$ is

$$
A(x)=\frac{c_{1}}{\tilde{\Delta}} \psi(\alpha, 0, x)
$$

and the function $\psi$ is defined in (28).

Proof By aid of composition rules, equation (12) can be rewritten as follows:

$$
D_{\pi,-}^{\alpha} p(x)^{C} D_{0,+}^{\alpha}\left[y_{\lambda}(x)+I_{0,+}^{\alpha} \frac{1}{p(x)} I_{\pi,-}^{\alpha} Y_{\lambda}(y)\right]=0 .
$$

The last equality implies that on the $C[0, \pi]$-space the function in brackets is a stationary function of the fractional Sturm-Liouville problem for Coulomb potential. $D_{\pi,-}^{\alpha} p(x)^{C} D_{0,+}^{\alpha}$, which according to (28) can be found as

$$
\begin{aligned}
\phi_{0} & =\xi_{1}+\xi_{2} I_{0,+}^{\alpha} \frac{(\pi-x)^{\alpha-1}}{\Gamma(\alpha) p(x)} \\
& =\xi_{1}+\xi_{2} \psi(\alpha, 0, x)
\end{aligned}
$$

equation (12) in the form of

$$
y_{\lambda}(x)+I_{0,+}^{\alpha} \frac{1}{p(x)} I_{\pi,-}^{\alpha} Y_{\lambda}(y)=\xi_{1}+\xi_{2} \psi(\alpha, 0, x) .
$$

To end the proof, we should connect coefficients $\xi_{j}$, $c_{j}$, values $j=1,2$ determining the boundary conditions (13), (14). Let us note that the following formula results from composition rules (9) and equation (31):

$$
I_{\pi,-}^{1-\alpha} p(x)^{C} D_{0,+}^{\alpha} y_{\lambda}(x)=-I_{\pi,-}^{1} Y_{\lambda}(y)+\xi_{2} .
$$


For a continuous function $y_{\lambda}$, we obtain the following values at the ends:

$$
\begin{aligned}
& \left.I_{\pi,-}^{1-\alpha} p(x)^{C} D_{0,+}^{\alpha} y_{\lambda}(x)\right|_{x=0}=-\int_{0}^{\pi} Y_{\lambda}(y) d x+\xi_{2}, \\
& \left.I_{\pi,-}^{1-\alpha} p(x)^{C} D_{0,+}^{\alpha} y_{\lambda}(x)\right|_{x=\pi}=\xi_{2} .
\end{aligned}
$$

Respectively, for $y_{\lambda}$, using (31) we find

$$
\begin{aligned}
y_{\lambda}(0) & =\phi_{0}(0)=\xi_{1}, \\
y_{\lambda}(\pi) & =\phi_{0}(\pi)-\left.I_{0,+}^{\alpha} \frac{1}{p(x)} I_{\pi,-}^{\alpha} Y_{\lambda}(y)\right|_{x=\pi} \\
& =\xi_{1}+\xi_{2} \psi(\alpha, 0, \pi)-\left.I_{0,+}^{\alpha} \frac{1}{p(x)} I_{\pi,-}^{\alpha} Y_{\lambda}(y)\right|_{x=\pi} .
\end{aligned}
$$

The following set of linear equations for coefficients $\xi_{j}$ results from (32)-(36):

$$
\begin{aligned}
& \xi_{1}=0, \\
& c_{1} \xi_{1}+\xi_{2}\left(-c_{2}+c_{1} \psi(\alpha, 0, \pi)\right)=c_{1} F,
\end{aligned}
$$

where $F=\left.I_{0,+}^{\alpha} \frac{1}{p(x)} I_{\pi,-}^{\alpha} Y_{\lambda}(y)\right|_{x=\pi}$.

Since $\tilde{\Delta} \neq 0$, the solution for coefficients $\xi_{j}(j=1,2)$ is unique:

$$
\begin{aligned}
& \xi_{1}=0, \\
& \xi_{2}=c_{1} F / \tilde{\Delta} .
\end{aligned}
$$

Substituting the above solution into (31), we recover equivalent integral equation (30).

Furthermore, we give notation such as

$$
\begin{aligned}
& m_{p}=\min _{x \in[0, \pi]}|p(x)|, \\
& A=\|A(x)\|, \quad M_{\varphi}=\|\varphi(x)\|,
\end{aligned}
$$

where $\|\cdot\|$ denotes the supremum norm on the space $C[0, \pi]$.

Theorem 12 Let $\alpha>1 / 2$. Suppose that $\tilde{\Delta} \neq 0, x \in(0, \pi]$. Then the unique continuous eigenfunction $y_{\lambda}$ for singular fractional Sturm-Liouville problem with Coulomb potential (12)(14) corresponding to each eigenvalue obeying

$$
\left\|\left(\frac{A}{x}+q(x)\right)+\lambda w_{\alpha}\right\|<\frac{m_{p}}{M_{\varphi}+A \varphi(\pi)}
$$

exists and such an eigenvalue is simple.

Proof We have to say that equation (30) can be interpreted as a fixed point condition on the function space $C[0, \pi]$,

$$
y_{\lambda}=T y_{\lambda},
$$


where the mapping on the right-hand side for any continuous function $g \in C[0, \pi]$ is defined as

$$
T g=-I_{0,+}^{\alpha} \frac{1}{p(x)} I_{\pi,-}^{\alpha} Y_{\lambda}(g)+\left.A(x)\left(I_{0,+}^{\alpha} \frac{1}{p(x)} I_{\pi,-}^{\alpha} Y_{\lambda}(g)\right)\right|_{x=\pi} .
$$

The following inequality will be useful in further estimations:

$$
\begin{aligned}
\left\|Y_{\lambda}(g)-Y_{\lambda}(r)\right\| & =\left\|\left(\left(\frac{A}{x}+q(x)\right) g+\lambda w_{\alpha} g\right)-\left(\left(\frac{A}{x}+q(x)\right) r+\lambda w_{\alpha} r\right)\right\| \\
& \leq\|g-r\|\left\|\left(\frac{A}{x}+q(x)\right)+\lambda w_{\alpha}\right\| .
\end{aligned}
$$

By performing necessary operations for the distance between images $\operatorname{Tg}$ and $\operatorname{Tr}$ for a pair of arbitrary continuous functions $g, r \in C[0, \pi]$,

$$
\begin{aligned}
\|T g-\operatorname{Tr}\| & =\left\|-I_{0,+}^{\alpha} \frac{1}{p(x)} I_{\pi,-}^{\alpha}\left(Y_{\lambda}(g)-Y_{\lambda}(r)\right)+A(x)\left(I_{0,+}^{\alpha} \frac{1}{p(x)} I_{\pi,-}^{\alpha}\left(Y_{\lambda}(g)-Y_{\lambda}(r)\right)\right)_{x=\pi}\right\| \\
& \leq\|g-r\| \cdot\left\|\left(\frac{A}{x}+q(x)\right)+\lambda w_{\alpha}\right\|\left(\frac{M_{\varphi}}{m_{p}}+\frac{A \varphi(\pi)}{m_{p}}\right) \\
& \leq\|g-r\| L
\end{aligned}
$$

where constant $L=\left\|\left(\frac{A}{x}+q(x)\right)+\lambda w_{\alpha}\right\|\left(\frac{M_{\varphi}}{m_{p}}+\frac{A \varphi(\pi)}{m_{p}}\right)$. By means of (37), we state that mapping $T$ is a contraction on the space $\langle C[0, \pi],\|\cdot\|\rangle$

$$
\|\operatorname{Tg}-\operatorname{Tr}\| \leq\|g-r\| L, \quad L \in(0, \pi)
$$

Hence, a unique fixed point enounced as $y_{\lambda} \in C[0, \pi]$ exists that solves equation (12), (30) and satisfies boundary conditions (13), (14) provided (37) is applied. In that case, such eigenvalues are simple. The proof is completed.

\section{Conclusion}

In the study, we focus on the spectral properties of the singular fractional Sturm-Liouville problem via Coulomb potential. We pointed that its eigenvalues related to the Coulomb potential with the certain boundary conditions are real and its eigenfunctions corresponding to distinct eigenvalues are orthogonal. We also prove that the fractional Sturm Liouville operator having Coulomb potential is self-adjoint. We give that some results of Sturm Liouville theory for fractional theory.

Competing interests

The authors declare that they have no competing interests.

Authors' contributions

All authors read and approved the final manuscript.

\section{Acknowledgements}

The authors sincerely thank the editor and reviewer for their valuable suggestions and useful comments that have led to the present improved version of the original manuscript. 


\section{References}

1. Hilfer, R (ed.): Applications of Fractional Calculus in Physics. World Scientific, Singapore (2000)

2. Carpinteri, A, Mainardi, F (eds.): Fractals and Fractional Calculus in Continuum Mechanics. Springer, Telos (1998)

3. West, BJ, Bologna, M, Grigolini, P: Physics of Fractal Operators. Springer, New York (2003)

4. Podlubny, I: Fractional Differential Equations. Academic Press, San Diego (1999)

5. Samko, SG, Kilbass, AA, Marichev, Ol: Fractional Integrals and Derivatives: Theory and Applications. Gordon and Breach, New York (1993)

6. Miller, KS, Ross, B: An Introduction to the Fractional Calculus and Fractional Differential Equations. Wiley, New York (1993)

7. Kilbas, AA, Srivastava, HM, Trujillo, JJ: Theory and Applications of Fractional Differential Equations. Elsevier, Amsterdam (2006)

8. Machado, JT, Kiryakova, V, Mainardi, F: Recent history of fractional calculus. Commun. Nonlinear Sci. Numer. Simul. (2010). doi:10.1016/j.cnsns

9. Baleanu, D, Og, M: On the existence interval for the initial value problem of fractional differential equation. Hacet. J. Math. Stat. 4(40), 581-587 (2011)

10. Eid, R, Muslih, SI, Baleanu, D, Rabei, E: On fractional Schrödinger equation in $\alpha$-dimensional fractional space. Nonlinear Anal. 10, 1299-1304 (2009)

11. Abolhassan, R, Baleanu, D, Vahid Johari, M: Conditional optimization problems: fractional order case. J. Optim. Theory Appl. 156(1), 45-55 (2013)

12. Guo-Cheng, W, Baleanu, D: New applications of the variational iteration method-from differential equations to q-fractional difference equations. Adv. Differ. Equ. 2013, 21 (2013). doi:10.1186/1687-1847

13. Baleanu, D, Octavian, GM, Agarwal, PR: Asymptotic integration of (1+alpha)-order fractional differential equations. Comput. Math. Appl. 62(3), 1492-1500 (2011)

14. Said Grace, R, Agarwal, RP, Wong, PJY, Zafer, A: On the oscillation of fractional differential equations. Fract. Calc. Appl. Anal. 15, 222-231 (2012)

15. Baş, E: Fundamental spectral theory of fractional singular Sturm-Liouville operator. J. Funct. Spaces Appl. 2013, Article ID 915830 (2013)

16. Klimek, M: On Solutions of Linear Fractional Differential Equations of a Variational Type Czestochowa. The Publishing Office of Czestochowa, University of Technology (2009)

17. Al-Mdallal, QM: An efficient method for solving fractional Sturm-Liouville problems. Chaos Solitons Fractals 40 183-189 (2009)

18. Erturk, VS: Computing eigenelements of Sturm-Liouville problems of fractional order via fractional differential transform method. Math. Comput. Appl. 16, 712-720 (2011)

19. Cabrera, IJ, Harjani, J, Sadarangani, KB: Existence and uniqueness of positive solutions for a singular fractional three-point boundary value problem. Abstr. Appl. Anal. 2012, 18 (2012)

20. Klimek, M, Argawal, OP: On a regular fractional Sturm-Liouville problem with derivatives of order in $(0,1)$. In 13 th Int Cont. Conf., 2012

21. Johnson, RS: An Introduction to Sturm-Liouville Theory. University of Newcastle, Upon Tyne Publishing (2006)

22. Zettl, A: Sturm-Liouville Theory. Mathematical Surveys and Monographs, vol. 121. Am. Math. Soc., Providence (2005)

23. Amrein, WO, Hinz, AM, Pearson, DB (eds.): Sturm-Liouville Theory, Past and Present. Birkhauser, Basel (2005)

24. Kreyszig, E: Advanced Engineering Mathematics, 9th edn. Wiley, Hoboken (2006)

25. Levitan, BM, Sargsjan, IS: Introduction to Spectral Theory: Self Adjoint Ordinary Differential Operators. Am. Math. Soc., Providence (1975)

26. Qi, J, Chen, S: Eigenvalue problems of the model from nonlocal continuum mechanics. J. Math. Phys. 52, 073516 (2011)

27. Panakhov, E, Bas, E: Inverse problem having special singularity type from two spectra. Tamsui Oxf. J. Inf. Math. Sci. 28(3), 239-258 (2012)

28. Panakhov, E, Sat, M: Reconstruction of potential function for Sturm-Liouville operator with Coulomb potential. Bound. Value Probl. 2013, 49 (2013)

29. Granas, A, Dugundji, J: Fixed Point Theory. Springer, New York (2003)

10.1186/1687-1847-2013-300

Cite this article as: Bas and Metin: Fractional singular Sturm-Liouville operator for Coulomb potential. Advances in

Difference Equations 2013, 2013:300

\section{Submit your manuscript to a SpringerOpen ${ }^{\circ}$ journal and benefit from:}

- Convenient online submission

- Rigorous peer review

- Immediate publication on acceptance

- Open access: articles freely available online

- High visibility within the field

- Retaining the copyright to your article 